\title{
Pulsar radio emission beams
}

\author{
Jarosław Kijak \\ J. Kepler Astronomical Center, Pedagogical University, Lubuska 2, \\ PL-65-265 Zielona Góra, Poland
}

\begin{abstract}
We study the structure of the pulsar beam using the analysis of beam radius. We indentify three emission cones in radio beams. The dependence of the beam radius on frequency is obtained.
\end{abstract}

\section{Introduction}

Two models of average pulsar beam structure appear in literature: nested-cones and patchy beam. Several studies present arguments for the multi-conal beam structure (Mitra \& Deshpande 1999; Gangadhara, Gupta, \& Lorimer, these proceedings). Moreover, as it was demonstrated by Gil \& Krawczyk (1996), patchy beam model is inconsistent with the observed frequency evolution of subpulses as contrasted with profile components.

In this work, we analyse beam radii at three frequencies using pulse widths from recently published papers (see References). We indentify three emission cones in radio beams. We also obtained the relationship on beam radius $\rho_{\nu}$.

\section{Analysis and Conclusions}

The opening angle $\rho$ of the pulsar beam corresponding to the pulse-width was calculated using the formula from Gil, Gronkowski, \& Rudnicki (1984). In our analysis, we used pulse widths at $10 \%$ of maximum intensity. Analysis of opening angles at several frequencies leads us to formulate the relationship for $\rho_{10}(\nu, P)$

$$
\rho=\left\{\begin{array}{ll}
6.4^{\circ} & \text { (outer) } \\
5.0^{\circ} & \text { (middle) } \\
3.6^{\circ} & \text { (inner) }
\end{array}\right\} \nu_{G H z}^{-0.09 \pm 0.03} P^{-0.5} .
$$

Mitra \& Deshpande (1999) analysed the separation between the peaks of the outermost conal components and confirmed existence of three emission cones. Our results are consistent with their conclusion. Finally, we argue that the emission beam has the multi-conal structure. The dependence of the beam radius $\rho$ on frequency $\nu$ is strong support to the radius-to-frequency mapping in pulsar emitting regions (cf. Kijak \& Gil 1998). 


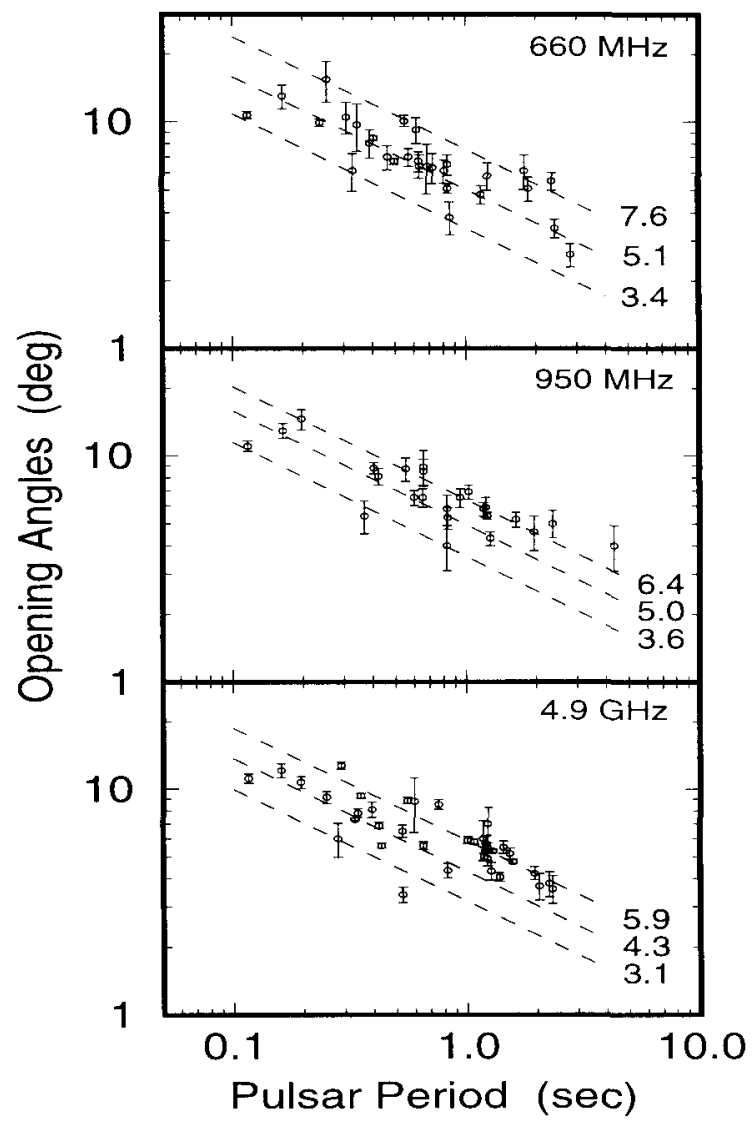

Figure 1. Analysis of opening angles $\rho$ at three frequencies. The dashed lines corresponding to different values of $A\left(\rho=A P^{-0.5}\right)$ reflect three emission cones. Formal weighted fits at $950 \mathrm{MHz}$ are given for three cones: (inner) $\rho=$ $\left(3.6^{\circ} \pm 0.6\right) P^{-0.42 \pm 0.4}$, (middle) $\rho=\left(5.0^{\circ} \pm 0.2\right) P^{-0.54 \pm 0.5}$, (outer) $\rho=\left(6.4^{\circ} \pm\right.$ $0.2) P^{-0.47 \pm 0.7}$, respectively.

Acknowledgments. This work was supported by KBN Grant 2 P03D 01512 .

\section{References}

Gil, J., Gronkowski, P., \& Rudnicki, W. 1984, A\&A, 132, 312

Gil, J., Krawczyk A., 1997, A\&A, 280, 143

Kijak, J. Gil, J. 1998, MNRAS, 299, 855

Kijak J., Kramer M., Wielebinski R., \& Jessner A. 1998, A\&A, 127, 153

Manchester, R. N, Han, J. L., \& Qiao, J. 1998, MNRAS, 295, 280

Mitra, D. \& Deshpande, A. A., 1999, A\&A, 346, 906

Ommen, T. D., D'Alessandro, F., Hamilton, P. A., \& McCulloch, P. M. 1997, 287, 307 